\title{
$\begin{array}{ll}\text { Research Square } & \text { Preprints are preliminary reports that have not undergone peer review. } \\ \text { They should not be considered conclusive, used to inform clinical practice, } \\ \text { or referenced by the media as validated information. }\end{array}$
}

\section{Comparative Analysis on Transcriptomics of Ivermectin Resistant and Sensitive Strains of Haemonchus Contortus}

\section{Waresi Tuersong}

Huazhong Agricultural University

\section{Caixian Zhou}

huazhong agricultural university

\section{Simin WU}

huazhong agricultural university

\section{Peixi Qin}

huazhong agricultural university

\section{Chunqun Wang}

huazhong agricultural university

\section{Wenda Di}

Guangxi university

lu liu

huazhong agricultural university

Hui liu

Huazhong agricultural university

Min Hu ( $\nabla$ mhu@mail.hzau.edu.cn )

Huazhong Agricultural University College of Plant Science and Technology https://orcid.org/00000001-8381-6328

\section{Research Article}

Keywords: Haemonchus contortus, Ivermectin, Resistance mechanism, Transcriptome analysis.

Posted Date: January 10th, 2022

DOI: https://doi.org/10.21203/rs.3.rs-1231250/v1

License: (1) (1) This work is licensed under a Creative Commons Attribution 4.0 International License.

Read Full License 


\section{Abstract}

Background: Ivermectin (IVM) is one of the most important and widely used anthelmintics in veterinary medicine. However, its efficacy is increasingly compromised by widespread resistance, and the exact mechanism of IVM resistance remains unclear for most parasitic nematodes including Haemonchus contortus, a blood-sucking parasitic nematode of small ruminants.

Methods: In this study, we isolated and assessed an IVM resistant strain from Zhaosu, Xinjiang, China. Subsequently, the comparative analyses on transcriptomics of IVM susceptible and resistant $H$. contortus adult worms were carried out using RNA sequencing and bioinformatics.

Results: In total, 543 and 359 differentially expressed genes (DEGs) were identified in male and female adult worms of the resistant strain compared with the susceptible strain, respectively. The DEGs encode molecules involved in receptor activities, transport, detoxification, lipid metabolism and cuticle collagen formation. In addition, Gene Ontology (GO) analysis revealed that transcriptional changes were dominant in genes associated with ligand-gated channel activity, oxidation-reduction process, lipid metabolic process, and structural constituent of cuticle. The results support previous proposal that the IVM resistant mechanism of $\mathrm{H}$. contortus involved in both neuromuscular and non-neuromuscular pathways. Finally, the quantitative RT-PCR results confirmed that the transcriptional profiles of selected DEGs (male: 8 genes, female: 10 genes) were consistent with those obtained by the RNA-Seq.

Conclusions: The findings from this work provided valuable information for further studies on the IVM resistance in $\mathrm{H}$. contortus.

\section{Background}

Haemonchus contortus is the most prevalent and pathogenic gastrointestinal nematode (GIN) of small ruminants, causing significant economic impacts on livestock production due to its high pathogenicity and widespread occurrence around the world [1]. Owing to the lack of effective vaccines and other control measures, three classes of anthelmintics (benzimidazoles, macrocyclic lactones and cholinergic agonists) are dominant in this parasite control. Among these, ivermectin (IVM) has been successful in the control of many parasites in both humans and animals for more than 30 years [2, 3]. However, as the uncontrolled use in livestock, the success is gradually undermined by the drug selection and spread of resistant parasite populations [4-6]. Consequently, its resistance has become widespread in many species of GIN of livestock.

Our knowledge about the resistant mechanisms of IVM has increased greatly over the last decades. Due to the technique limitations, the initial research on the IVM resistant mechanism mainly focused on the polymorphisms and expression level of candidate genes encoding the target receptors, such as $\mathrm{Hc}-\mathrm{lgc}-37$, $\mathrm{Hc}-\mathrm{g} / \mathrm{c}-3, \mathrm{Hc}-\mathrm{g} / \mathrm{c}-5$ and Hc-avr-14 [7-9]. Subsequently, attention turns to observing differences (polymorphisms and expression level) between resistant and susceptible strains or pre- and posttreatment in the P-gps and dyf-7 [10-13]. However, due to the extremely high levels of genetic diversity 
among $H$. contortus populations and lack of consistency between studies [14-16], the major genetic mediators of IVM resistance have not been unequivocally identified.

Fortunately, the high-quality reference genome of this parasite together with the increasing accessibility to high throughput RNA sequencing offers an opportunity to facilitate aimed studies to explore its resistant mechanism. Transcriptome sequencing has been used widely to identify candidate genes/pathways related to drug resistance $[17,18]$. However, the transcriptome changes of $H$. contortus IVM field resistant strain induced by natural selection, have received little attention. In the present study, to explore transcriptomic difference and identify the candidate genes potentially related with drug resistance induced by long term selection of IVM in the field sample, the $H$. contortus transcriptome of adult worms were sequenced and a comparative analysis was performed between IVM susceptible and field resistant strains. The identified differentially expressed genes (DGEs) and subsequent enrichment analysis provided a theoretical basis for further understanding of IVM resistance in $\mathrm{H}$. contortus.

\section{Methods}

\section{ivermectin resistance and susceptible strains}

1. Susceptible strain: Haecon-5 strain kindly presented by Professor Robin B. Gasser (University of Melbourne) and maintained in goats in Huazhong Agricultural University.

2. Resistance strain: isolated from Zhaosu (Xinjiang, China) and maintained in goats in Huazhong Agricultural University.

\section{The control test and faecal egg count reduction test (FECRT)}

In order to further confirm the IVM resistance of Zhaosu strain, six goats (6-months old goats free of parasites) were infected with $H$. contortus of Zhaosu strain (7000 L3s per goat) and randomly divided into treated and untreated groups (three animals per group). The goats in treatment group were treated with $0.2 \mathrm{mg} / \mathrm{kg}$ (with sub-cutaneous injection) IVM thirty days after infection, and all animals were necropsied 14 days post-treatment and $H$. contortus worm burdens were counted. Resistance was confirmed when the reduction in mean worm counts was less than $90 \%(R(\%)=(1-[E / C]) \times 100$ where $E$ and $C$ represent mean worm burden of treated and un-treated group) [19].

In addition, the FECRT was conducted based on the McMaster method. In brief, $2 \mathrm{~g}$ of individual faecal samples were homogenised in $58 \mathrm{ml}$ of saturated sodium nitrate. Then, $0.15 \mathrm{ml}$ of the suspension was added to each chamber of the slide for egg count after $5 \mathrm{~min}$. The eggs per gram (EPG) was calculated by multiplying the number of eggs in the two chambers by 100 . The FEC reduction was calculated according to the formula FECR $(\%)=100 \times(1-[\mathrm{Xt} / \mathrm{Xc}])$ where $\mathrm{c}$ and $\mathrm{t}$ mean the average of EPG for untreated and treated group, respectively, at 14 day post treatment, and resistance is confirmed when FECR is $<95 \%$, and the $95 \%$ confidence level is less than $90 \%$ [20]. 


\section{Larval development assay (LDA)}

Fresh eggs were collected from the faeces based on a previous study [21]. LDA was carried out as modified from a previous study [22]. Briefly, the $2.4 \mathrm{ml}$ suspension (2 $\mathrm{ml}$ egg suspension and $400 \mu \mathrm{l}$ growth medium, $\sim 5000$ eggs) was added to T25 cell bottle and incubated at $27^{\circ} \mathrm{C}$ for $24 \mathrm{~h}$ for the hatching of the first-stage larvae (L1). On the next day, the L1 larval suspension ( $99 \mu \mathrm{l}, \sim 100$ larvae) was aliquoted into the 96-well plates and $1 \mu$ IVM working solution was added to each well and then the L1s were incubated at $27^{\circ} \mathrm{C}$ for another 6 days. IVM concentrations ranged from $12.5 \mathrm{ng} / \mathrm{ml}$ to $0.2 \mathrm{ng} / \mathrm{ml}$ for Zhaosu strain and $5 \mathrm{ng} / \mathrm{ml}$ to $0.1 \mathrm{ng} / \mathrm{ml}$ for Haecon- 5 strain. The number of developed third-stage larvae (L3s) was counted and the L3 developmental rate was expressed as a percentage of the mean number in control wells. Three separate assays with triplicate were conducted for each concentration. The data were analysed using GraphPad Prism 8 software. Resistance ratio (RR) was calculated according to the formula: IC50 resistant isolate/IC50 susceptible isolate [23].

\section{Sample preparation}

The adult male and female worms of $H$. contortus were isolated from the abomasum of goats 30 days after infection with 7000 L3 of either Haecon-5 strain or Zhaosu strain. These worms were washed thoroughly in PBS and randomly assigned into four groups of 15 worms each with three biological replicates (resistance males, susceptible males, resistance females, susceptible females). All samples transferred to liquid nitrogen for storage until use.

\section{RNA extraction, library preparation and sequencing}

Total RNA was extracted using TRIzol® Reagent with manufacturer's instructions (Invitrogen, USA). RNA integrity was precisely assessed using 2100 Bio-analyzer (Agilent Technologies USA), and RNA samples with RIN $\geq 8$ were used to construct library. The transcriptome libraries were prepared using Illumina kit (San Diego, CA, USA) following the manufacturer's instructions. Briefly, polyA mRNA was isolated using oligo(dT) beads and cleaved in fragmentation buffer. Then, cDNA was synthesized using a SuperScript dscDNA synthesis kit (Invitrogen, CA) with random hexamer primers (Illumina). After quantified, the library was sequenced with the Illumina NovaSeq 6000 sequencer (Majorbio, Shanghai, China). The samples were named as RM1, RM2, RM3; RF1, RF2, RF3; SM1, SM2, SM3; SF1, SF2, SF3, respectively. The RNA-seq raw data were submitted to the National Centre for Biotechnology Information (NCBI) Sequence Read Archive with a BioProject ID: PRJNA772807.

\section{Quality control and read mapping}

The sequence quality of raw reads was controlled with SeqPrep (https://github.com/jstjohn/SeqPrep) and Sickle (https://github.com/najoshi/sickle). After removing low quality reads, the Q20, Q30, and GC were calculated, and clean reads were mapped to the reference $H$. contortus genome (https://parasite.wormbase.org/Haemonchus_contortus_prjeb506/Info/Index/) using software HISAT2 (https://ccb.jhu.edu/software/hisat2/ index.shtml) and assembled by StringTie (https://ccb.jhu.edu/software/stringtie/index.shtml?t=example). 


\section{Differential gene expression (DGE) and enrichment analysis}

The gene expression level was normalized by using transcripts per million reads (TPM), and expression analysis was carried out using RSEM (http://deweylab.github.io/RSEM/). The differently expressed genes (DEGs) were identified with $|\log 2 \mathrm{FC}|>1$ and padj<0.05 using DEseq2

(http://bioconductor.org/packages/stats/bioc/DESeq2/). The venn, heat map clustering, and volcano diagrams were drawn by $\mathrm{R}$ packages.

To better evaluate the potential roles of the DEGs, the functional analysis of the DEGs was carried out based on the Gene Ontology (GO) and Kyoto Encyclopedia of Genes and Genomes (KEGG). GO and KEGG pathway enrichment analyses were performed via Majorbio cloud platform (https://cloud.majorbio.com/).

\section{Validation of transcriptome sequencing results}

Eleven DEGs were selected and primers designed using Primer-BLAST (Additional file 1: Table S1). Total RNA from the resistant adult male worms (RM), resistant adult female worms (RF), susceptible adult male worms (SM) and susceptible adult female worms (SF) was reverse-transcribed to the CDNA with HiScript II Q RT SuperMix (Vazyme, China). The qRT-PCR was conducted to assess mRNA levels of selected DEGs in biological triplicates with technical duplicates using a TB Green ${ }^{\circledR}$ Premix Ex Taq ${ }^{\mathrm{TM}}$ II (Takara, China). The reaction procedure: $95^{\circ} \mathrm{C}$ for $5 \mathrm{~min}, 40$ cycles at $95^{\circ} \mathrm{C}$ for $15 \mathrm{~s}, 60^{\circ} \mathrm{C}$ for $30 \mathrm{~s}$ and $72^{\circ} \mathrm{C}$ for $15 \mathrm{~s}$. The actin gene was used as an endogenous reference gene (Gene ID: DQ080917) [24], and relative expression fold change of each gene in resistant samples versus susceptible ones was calculated using the $2^{-\Delta \Delta C t}$ method.

\section{Statistical analysis}

All experiments were independently conducted three times and analysed by GraphPad Prism 8.0.2 (GraphPad Company, USA). The t-test was performed to compare DEGs in resistant and susceptible strains and the results were defined significant as $P<0.05$ ( ${ }^{*} P<0.05$, ${ }^{\star *} P<0.01, * \star * P<0.001$, ${ }^{\star \star \star *} P<$ 0.0001).

\section{Results}

Detection of IVM resistance in $\mathrm{H}$. contortus isolated from Zhaosu

\section{The control test and faecal egg count reduction test (FECRT)}

The control and FECRT tests were carried out using $0.2 \mathrm{mg} / \mathrm{kg}$ of IVM in the treatment to assess IVM efficacy, respectively. The result of control test revealed that the percentage reduction in worm burdens was $32.5 \%$ (Table 1). In addition, the result of FECRT showed that the EPGs in treatment group were not 
decreased significantly at day 14 post treatment compared with the un-treatment group and the FECR was $1 \%$ in the IVM administration group (Table 2 ).

\section{Table 1}

Haemonchus contortus worm burdens in IVM treatment and control groups

\begin{tabular}{|lcccccc|}
\hline & C1 & C2 & C3 & E1 & E2 & E3 \\
\hline Male & 1360 & 371 & 131 & 1069 & 195 & 160 \\
\hline Female & 1793 & 473 & 402 & 1172 & 250 & 210 \\
\hline Total & 3153 & 844 & 533 & 2241 & 445 & 370 \\
\hline Mean worm burden & 1510 & & & 3056 & & \\
\hline Reduction (\%) & 32.5 & & & & & \\
\hline C and E represent mean worm burden of un-treatment and treatment group. \\
\hline
\end{tabular}

Table 2

Faecal egg count reduction rate (FECR\%), 95\% confidence intervals (Upper $\mathrm{Cl} \%$ and Lower $\mathrm{Cl}$ $\%)$ and resistance state of Haemonchus contoruts against ivermectin

\begin{tabular}{|lll|}
\hline & Faecal egg counts at 14 days post treatment (EPG:eggs per grams) \\
\hline & Untreatment group & Treatment group \\
\hline Mean EPG & 6200 & 6167 \\
\hline FECR (\%) & & 1 \\
\hline Upper $95 \% \mathrm{Cl}$ & 89 \\
Lower $95 \% \mathrm{Cl}$ & 0 \\
Drug effectiveness & Resistant \\
\hline
\end{tabular}

\section{Larval developmental assay (LDA)}

The results of LDA showed that with the increasing of the drug concentrations, larval developmental rate decreased and dropped to zero at the highest IVM concentration for each group (Fig. 1). The IC50 values were $0.218 \mathrm{ng} / \mathrm{ml}(95 \% \mathrm{Cl}: 0.208$ to 0.227$)$ and $1.291 \mathrm{ng} / \mathrm{ml}(95 \% \mathrm{Cl}: 1.209$ to 1.377) for Haecon-5 and Zhaosu-R, respectively and the RR was calculated as 5.9 .

\section{Transcriptome sequencing and data assembly}

The total raw reads were generated from resistant and susceptible adult male and female worm transcriptomes (four groups) with three replicates for each sample, respectively. After quality control, a total of $93.98 \mathrm{~Gb}$ of clean data were obtained from the twelve samples altogether, each of which 
contained more than $7.19 \mathrm{~Gb}$ with quality score of Q20 $\geq 98.35$ and Q30 $\geq 94.71$, indicating high-quality sequencing (Additional file 1: Table S2). A range of 74.6-78.36\% clean reads of each sample were aligned onto $H$. contortus reference genome (Additional file 1: Table S3). Principal component analysis (PCA) of the normalized RNA-seq read counts showed a high level of consistency and good separation between biological replicates of the same population from susceptible or resistant strains (Fig. 2). A total of 17857 genes were identified and annotated from the four groups, and 14083 (known: 12898, novel: 1185), 12153 (known: 11266, novel: 887), 14144 (known: 12931, novel: 1213), 12405 (known: 11487, novel: 918$)$ genes were detected in RM, RF, SM and SF, respectively.

\section{Differential gene expression analysis}

In this study, the differentially expressed genes (DEGs) were identified by the comparison on the changes in transcriptome profiles of male or female worms between $H$. contortus resistant and susceptible strains. 543 and 359 DEGs were identified in male (RM vs RS) and female (RF vs SF) comparisons, respectively (Fig. 3b, c). Among them, 429 DEGs were male-specific whereas 245 DEGs were female-specific, and 114 DEGs were present in both male and female groups (Fig. 3a). In RM, 338 genes were up-regulated and only 205 down-regulated, whereas in RF, 105 up-regulated and 254 down-regulated. All DEGs were used for hierarchical cluster analysis (Fig. 4a, b), showing that DGEs from the biological replicates were more closely clustered together. In addition, top ten up and down regulated genes in each comparison group were identified (Additional file 1: Table S4), containing many un-characterised genes ( 5 up and 7 down for $\mathrm{RM}, 2$ up and 5 down for RF) and those with known or putative functions including parasitic stage specific protein 1, RNA-directed DNA polymerase (Reverse transcriptase) domain containing protein, saposin type B domain containing protein, glutathione S-transferase, cytoplasmic dynein 2 light intermediate chain 1 and nematode cuticle collagen. In addition, among the DEGs identified in the transcriptomes, genes with functions involved in receptor activities, transport, detoxification, structural constituent of cuticle, lipid binding, transport, metabolism and signalling pathway were also identified.

\section{DEGs encoding receptors}

Early reports highlighted that IVM can interact with a wider range of ligand-gated channels found in parasitic nematodes, including GluClRs, $y$-aminobutyric acid (GABA), nicotinic acetylcholine (nAChR) and glycine (Gly) receptors (Laing et al., 2017). Interestingly, among the DEGs identified in the present study, the GluClRs subunits encoding gene g/c-5 (HCON_00161180) was down-regulated (log2 fold-change: -4.05) in RF. In addition, the genes that encoding the acetylcholine receptor were up and down regulated in the RM (up:2 down:4) and RF (up:1 down:1), and the G protein-coupled receptor (GPCR) genes were also up and down regulated in both RM (up:5 down:1) and RF (up:1 down:4) as compared with SM and SF (Additional file 1: Table S5).

\section{DEGs involved in detoxification and transport}

Except for the receptor encoding genes, 13 genes encoding molecules involved in detoxification including cytochromes P450 (CYPs), short-chain dehydrogenases/reductases (SDR), glutathione S-transferases 
(GSTs), and UDP-glycosyltransferases (UGTs), were up or down regulated (Additional file 1: Table S5). A total of four and two CYP450 encoding genes were found up-regulated in RM and RF, respectively. Two SDR genes were only up regulated in RM and three GST genes were down regulated both in RM and RF, respectively. One UGT gene was up regulated both in RM, another one and two UGT genes were up and down regulated in $\mathrm{RF}$, respectively. In addition, the transcription level of the $A B C$ transporter encoding gene P-gp-9.1 (HCON_00130050) was significantly higher in both RM and RF (log2 fold-change: 2.20 and 1.2, respectively), and $\mathrm{Hco}$-abt-4 (HCON_00085890, log2 fold-change: 1.18) was significantly up-regulated in RF (Additional file 1: Table S5).

\section{DEGs involved in lipid metabolism and cuticle collagen formation}

Beyond mentioned above, most notable is that genes related with lipid metabolic process, cellular lipid metabolic process, lipid binding, fatty acid metabolic process, fatty acid biosynthetic process showed significantly up-regulated in RM (11 up-regulated) and RF (5 up-regulated) (Additional file: Table S6). In addition, 23 and 17 cuticle collagen genes showed differential expression in RM and RF, respectively, of which 21 (RM) and 15 (RF) were significantly down-regulated (Additional file 1: Table S6).

\section{GO enrichment analysis of the DEGs}

For functional annotation and classification, the functions of DEGs were evaluated using GO enrichment analysis and the identified DEGs were assigned GO IDs and divided into different functional groups, which belong to three main categories: biological processes (BP), cellular components (CC), and molecular functions (MF). In the male worms, up-regulated DEGs (Fig. 5a) significantly enriched in intrinsic component of membrane (GO: 0031224), integral component of membrane (GO: 0016021), peptidase activity (G0:0008233), endopeptidase activity, oxidoreductase activity (G0:0016491), proteolysis (GO:0006508), oxidation-reduction process (GO:0055114), lipid metabolic process (G0:0006508). In contrast, down-regulated DEGs (Fig. 5b) significantly enriched in cellular component, intrinsic component of membrane, integral component of membrane, structural constituent of cuticle (G0:0042302), ligand-gated channel activity (GO:0022834), G protein-coupled receptor signaling pathway (GO:0007187), respectively.

In the female worms, up-regulated DEGs (Fig. 5c) significantly enriched in cellular component (G0:0005575), cellular anatomical entity (G0:0110165), intrinsic component of membrane (GO: 0031224), integral component of membrane (GO: 0016021), oxidoreductase activity (G0:0016491), oxidation-reduction process (GO: 0055114), lipid metabolic process (GO:0006508), cellular lipid metabolic process (G0:0044255). In contrast, down-regulated DEGs (Fig. 5d) significantly enriched in cellular component (GO:0005575), intrinsic component of membrane (GO: 0031224), integral component of membrane (GO: 0016021), protein-containing complex (G0:0032991), membrane (G0:0016020), peptidase activity (GO:0008233), transporter activity (G0:0005215), transmembrane transporter activity (GO:0022857), structural constituent of cuticle (G0:0042302), cation transmembrane transporter activity (GO:0008324), proteolysis (GO:0006508), transmembrane transporter (GO:0055085), ion transport 
(G0:0006811). In general, the GO term enrichment of the DEGs for the male and female showed a similar list of terms, especially in related with oxidation-reduction process, lipids and cuticle.

\section{KEGG functional annotation of the DEGs}

The ingenuity pathway analysis identified 79 (up-regulated DEGs) and 55 (down-regulated DEGs) for male, 96 (up-regulated DEGs) and 129 (down-regulated DEGs) pathways for female DEGs in resistant strain compared with susceptible strain SM and SF, respectively. The up-regulated DEGs related to KEGG pathway significantly enriched in autophagy-animal, lysosome, apoptosis, sphingolipid signalling pathway and $A B C$ transporters in the male (Fig. 6a). The down-regulated DEGs significantly enriched in lysosome, autophagy-animal, apoptosis, antigen processing and presentation and ECM-receptor interaction (Fig. 6b).

In the female (Fig. 6), the up-regulated DEGs significantly enriched in nicotine addiction, central carbon metabolism in cancer, $A B C$ transporters (Fig. 6c). The down-regulated DEGs significantly enriched in lysosome, apoptosis, sphingolipid signalling pathway, drug metabolism - cytochrome P450 (Fig. 6d). In general, the major enriched pathways for the male and female also showed a similar pattern in some pathway.

\section{qRT-PCR validation}

To further validate transcriptome result, eight (male) and ten (female) DEGs were selected for qRT-PCR analysis in adult male and female worms of $H$. contortus resistant and susceptible strains. As expected, the transcription results of selected genes obtained using qRT-PCR were in agreements with the RNA-seq results (male, t test: HCON_00013510, $p=0.0246$; HCON_00191400, $p=0.0729$; HCON_00130050, $p=0.035$; HCON_00130390, $p=0.0034$; HCON_00157290, $p<0.0001$; HCON_00192750, $p=0.0003$; HCON_00087240, $p<0.0001$; HCON_00007280 $p=0.0011$; female, $t$ test: HCON_00013510, $p=0.0001$; HCON_00191400, $p=0.0383 ;$ HCON_00130050, $p=0.0023$; HCON_00130390, $p=0.0036$; HCON_00157290, $p<0.0001$; HCON_00007280, $p=0.0004$; HCON_00003380, $p<0.0001$; HCON_00003390, $p<0.0001$; HCON_00162030, $p<0.0001 ;$ HCON_00192750, $p=0.0007)$. Overall, the RNA-seq data were well supported by qRT-PCR results (Fig. 7a, b).

\section{Discussion}

As $H$. contortus has shown a strong ability to develop resistance, IVM resistance in this parasite from different continents has been extensively studied and well documented [25-31]. However, our understanding of the molecular basis of the resistant mechanism is patchy. With advances in the application of genomic and transcriptomic technologies in exploring the mechanism of anthelmintic resistance, more recent studies focus on analysing the polymorphisms and expression level of the candidate genes. Therefore, in order to further understanding mechanism of IVM resistance in $H$. contortus, we carried out comparative transcriptome analysis between $\mathrm{H}$. contortus adult worms of susceptible (Haecon-5) and resistant (Zhaosu) strains using RNA-Seq. In the present study, a total of 543 and 359 DEGs were identified in the RM vs SM (up: 338, down: 205) and RF vs SF (up: 105, down: 254), 
respectively. Notably, although both sexes have the same selection pressure, the amount of DEGs in the $\mathrm{RM}$ was significantly higher than in the RF, and more DEGs are up-regulated in the RM, but it is opposite in the SF. Considering IVM treatment causing the dysregulation of the genes expression related with embryo development in nematodes [32], these differences may be related with the effects of IVM on the eggs in utero of the female worms. Furthermore, these differences may also be related to the different mechanism of IVM resistance between male and female worms, and the reasons hopefully can be identified in the future when more data from field resistant strains are available.

In previous studies, GluCIRs gene families often considered to be involved in IVM resistance in $H$. contortus. For example, it has reported that haplotype frequency and transcription level changes of $\mathrm{Hco}$ glc-5 may be related with IVM resistance [33, 34]. Furthermore, the Hco-glc-5 was also down-regulated in female worms of both the MHco4(WRS) and MHco10(CAVR) resistant strains comparing with the IVMsusceptible strain [35]. Hence, it was expected that long term selection would lead to transcriptional changes of GluClR genes in the resistant strain. As expected, the transcription level of Hco-glc-5 was significantly reduced in RF. However, recent studies also found that there are no significant differences in loci of $\mathrm{Hc}-\mathrm{g} / \mathrm{c}-5$ between IVM-resistant and sensitive isolates and no evidence of introgression in either backcross $[15,16]$. Taken together, the inconsistent results from different studies may be caused by differences in the genetic background of different strains, and suggest that $\mathrm{Hc}-\mathrm{g} / \mathrm{c}-5$ may be related to IVM resistance in some strains, but more powerful evidence is required to define whether $\mathrm{Hc}-\mathrm{g} / \mathrm{c}-5$ to be major gene conferring IVM resistance.

Although IVM is often considered to only act on different $\mathrm{GluCl}$ channels of nematodes, intriguingly, there are growing evidences that it also acts on other ion channels [36]. For example, in the free-living nematode Caenorabditis elegans, recent studies demonstrated that IVM inhibits $C$. elegans muscle LAChR receptors [37] and abamectin acts more complex antagonistic effect on nAChRs [38]. In $H$. contortus, a recent study identified 26 candidate genes in relation to $H$. contortus IVM resistance including nicotinic acetylcholine receptors (nAChRs) and G protein-coupled receptors (GPCRs) encoding genes [39]. In another study, several nAChRs and GPCRs genes were also up and down-regulated in both resistant strains [35]. In the present study, seven nAChRs genes and ten GPCR genes were differentially regulated in the RM and RF, respectively. Among these genes, Hco-acr-6 (HCON_0016650, predicted to be involved in cell communication, nervous system process, regulation of membrane potential and located in synapse.) was up-regulated in both RM and RF, which is in agreements with a previous report (Hco-acr6 upregulated in IVM-resistant strain (MHco10)) [35]. This gene may be one of the important candidate genes given it is a member of ligand-gated ion channel gene families. Meanwhile, down-regulated GO terms include ligand-gated channel activity, G protein-coupled receptor signalling pathway, ion transport and ion transmembrane transporter activity, implying that IVM may also have a significant effect on nAChRs and GPCRs. However, due to the complexity of two receptor families, it is a considerable challenge for studying modes of IVM action on these receptors, and further functional validation is required. 
In $H$. contortus, it has been reported that an up-regulation of $A B C$ transporters had a direct correlation with IVM resistant phenotype [40] and the final concentration of IVM in $H$. contortus [41]. In this study, P-gp-9.1 was significantly up-regulated in both RM and RF, and $A B C$ transport pathway enriched in KEGG analysis (Fig. 6a, c). These results are consistent with a role for ABC transporters in IVM susceptibility in $H$. contortus, implying that $\mathrm{ABC}$ transporters (particularly P-gp) clearly contribute to IVM efflux in field resistant strain. Together, these results raise the possibility that P-gp could be a potential component of a set of diagnostic tools for the detection of IVM-resistance in $H$. contortus. However, due to the multigenic nature of IVM resistance, more field resistant strains are required to be sequenced to confirm the suitability.

$H$. contortus is armed with many metabolizing enzymes (42 CYPs, 44 SDR, 34 UGT and 28 GST) to protect against environmental toxins and anthelmintics [42]. The previous study showed that silencing the gene CYP (HCON_00143950) increased the sensitivity to IVM in larvae of $H$. contortus [39]. In the adult worms of this parasite, although IVM induced changes in the expression of CYPs [43], it can't be metabolized by xenobiotic-metabolizing enzymes (XME) [44]. In the present study, CYPs and SDR were up-regulated and GST was down-regulated in the resistant strain, whereas UGT was both up- and downregulated. At first sight, these upregulated genes could be elucidated as being part of candidate genes that metabolize the IVM. Yet the UGTs and GSTs were downregulated which expected to be up-regulated in resistant strain. In $C$. elegans, RNA interference (RNAi) caused phenotypes of nine CYP genes associated with the embryonic lethality, uncoordinated movement, slow and stunted growth, and reduced fat storage [45]. Furthermore, CYPs were considered involved in fatty acid metabolism after exposure to IVM in the $C$. elegans IVM resistant strain [46]. Interestingly, in the present study, up-regulated XME genes were mainly involved in oxidation-reduction process, iron ion binding, heme binding, and up-regulated DEGs were mainly enriched in oxidation-reduction, lipid metabolic process, cellular lipid metabolic process, fatty acid metabolic process. These results suggest that the upregulated XME genes may play a role in detoxify endogenously accumulated metabolic compounds, such as lipid metabolism.

Lipids play indispensable roles in many aspects of intra- or inter-cellular signalling, cellular membranes, energy storage in the organisms [47]. The recent research reported that lipids modulate the activity and expression of efflux pumps, contributing to the development of the resistance in cancer $[48,49]$. In addition, the dramatic changes after exposure to IVM were dominated by genes related with lipid metabolism (C. elegans, resistant strain) and rapidly consumption of lipid stores (Globodera pallida) [46, 50]. Intriguingly, in the present study, genes related with lipids and lipid metabolism underwent the greatest change in the up-regulated terms, include lipid metabolic process, fatty acid biosynthetic process, fatty acid metabolic process, lipase binding and activity, cellular lipid metabolic process, glycerolipid metabolic process terms (Fig. 5a, c). These great difference in energy homeostasis between the susceptible and resistant strains suggest that IVM has multiple effects involving both neuromuscular and non-neuromuscular targets. These up-regulated genes likely play key roles in nutritional requirements, anti-oxidation, membrane fluidity, physical rigidity of the worm cuticle, to protect the worm itself from drug stimulation and adverse environment. To sum up, lipids and its metabolic pathways may play 
important roles in the IVM resistance, although it remains to be verified whether the lipids play a role in IVM-induced stress response, or direct role in IVM metabolism.

The cuticle is an external structure in all nematodes which plays a critical role in maintenance of body morphology and integrity, and locomotion [51]. Previous studies showed that the dysregulation of collagen encoding gene was considered to likely represent a non-specific marker of stress, such as oxidation, response to multiple pathogens and drugs [51-54]. Furthermore, in C. elegans, it was found that trans-cuticular absorption may be a mode of entry for anti-nematodal IVM [55] and that dye-filling (dyf) mutations appear to affect cuticle permeability of IVM and the dye [56]. In addition, recent study reported that $H$. contortus can absorb nutrients and some anthelmintics (levamisole and macrocyclic lactones) through the cuticle [57]. In B. malayi, 17 out of 29 cuticle collagen genes were significantly down-regulated after exposure to IVM [32]. In the present study, 23 and 17 cuticle collagen genes displayed differential expression in both RM and RF, of which 21 and 14 were significantly downregulated (Table S6), respectively. In the GO enrichment analysis, collagen trimer and structural constituent of cuticle were significantly enriched in both RM and RF (Fig. 5b, d). These down-regulated genes play key roles the morphological changes in the integrity of the worm cuticle, and likely involved in protect the worm itself from IVM stimulation. These results suggest that the trans-cuticular penetration may be an important mode of IVM entry into worms and may represent novel candidate pathway for IVM resistant mechanism in the parasitic nematodes. The long-term IVM stimulation

\section{Conclusions}

In present study, we made comparative analyses on the transcriptome profiles between IVM resistant and susceptible strains of $H$. contortus. In addition to some candidate genes, we also found some DEGs related with nAChR, GPCRs, lipid metabolism and cuticle morphological formation. Our results suggested that there are different mechanisms in different populations and IVM resistance may be selected by changes of $H$. contortus metabolism. Our findings provided new information for further research on the molecular mechanisms of the response to IVM in parasitic nematodes.

\section{Declarations}

\section{Acknowledgements}

Not applicable

\section{Funding}

This study was supported by the "National Natural Science Foundation of China" (Grant No. 31872462 and 32172881) to M.H.

\section{Availability of data and materials}


The datasets of this article are included within the manuscript and its supplementary material. All the RNA-seq raw data were submitted to the National Centre for Biotechnology Information (NCBI) Sequence Read Archive with a BioProject ID: PRJNA772807.

\section{Authors' contributions}

M.H. conceived, designed the experiments, obtained the fundings and revised the manuscript. T.W. and S.M.W. carried out the experiments (LDA, the controlled test and FECRT). T.W. and C.X.Z. analysed the sequencing data. T.W. and P.X.Q. carried out the experiments (qPCR). C.Q.W, W.D.D., L.L., H.L. contributed to the materials. T.W. wrote an initial version of the manuscript.

\section{Ethics approval and consent to participate}

Helminth-free goats were maintained in accordance with protocols approved by the Scientific Ethic Committee of Huazhong Agricultural University (Permit code: HZAUGO-2016-007) and Animal Ethics Guidelines from the People's Republic of China.

\section{Consent for publication}

Not applicable.

\section{Competing interests}

The authors declare that there is no competing interest.

\section{Author details}

${ }^{1}$ State Key Laboratory of Agricultural Microbiology, Key Laboratory for the Development of Veterinary Products, Ministry of Agriculture, College of Veterinary Medicine, Huazhong Agricultural University, Wuhan 430070, Hubei, China

${ }^{2}$ College of Animal Science and Technology, Guangxi University, Nanning 530004, Guangxi, China.

\section{References}

1. Emery DL, Hunt PW, Le Jambre LF. Haemonchus contortus: the then and now, and where to from here? Int J Parasitol. 2016;46:755-69.

2. Omura S, Crump A. The life and times of ivermectin- a success story. Nat Rev Microbil. 2004;2(12):984-9.

3. Fox LM. Ivermectin: uses and impact 20 years on. Curr Opin Infect Dis. 2006;19:588-93.

4. Kaplan RM, Vidyashankar AN. An inconvenient truth: global worming and anthelmintic resistance. Vet Parasitol. 2012;186:70-8.

5. Redman E, Sargison N, Whitelaw F, Jackson F, Morrison A, Bartley DJ, et al. Introgression of ivermectin resistance genes into a susceptible Haemonchus contortus strain by multiple backcrossing. PLoS Pathog. 2012;8:e1002534. 
6. Wang C, Li F, Zhang Z, Yang X, Ahmad AA, Li X, et al. Recent research progress in China on Haemonchus contortus. Front Microbiol. 2017;8:1509.

7. Blackhall WJ, Pouliot JF, Prichard RK, Beech RN. Haemonchus contortus: selection at a glutamategated chloride channel gene in ivermectin- and moxidectin-selected strains. Exp Parasitol. 1998;90:42-8.

8. Williamson SM, Wolstenholme AJ. P-glycoproteins of Haemonchus contortus. development of realtime PCR assays for gene expression studies. J Helminthol. 2012;86:202-8.

9. Kotze AC, Hunt PW, Skuce P, von Samson-Himmelstjerna G, Martin RJ, Sager H, et al. Recent advances in candidate-gene and whole-genome approaches to the discovery of anthelmintic resistance markers and the description of drug/receptor interactions. Int J Parasitol Drugs Drug Resist. 2014;4:164-84.

10. Raza A, Kopp SR, Bagnall NH, Jabbar A, Kotze AC. Effects of in vitro exposure to ivermectin and levamisole on the expression patterns of $A B C$ transporters in Haemonchus contortus larvae. Int $J$ Parasitol Drugs Drug Resist. 2016;6:103-15.

11. Maté L, Ballent M, Cantón C, Ceballos L, Lifschitz A, Lanusse C, et al. Assessment of P-glycoprotein gene expression in adult stage of Haemonchus contortus in vivo exposed to ivermectin. Vet Parasitol. 2018;264:1-7.

12. Kellerová P, Matoušková P, Lamka J, Vokřál I, Szotáková B, Zajíčková M, et al. Ivermectin-induced changes in the expression of cytochromes P450 and efflux transporters in Haemonchus contortus female and male adults. Vet Parasitol. 2019;273:24-31.

13. Urdaneta-Marquez L, Bae SH, Janukavicius P, Beech R, Dent J, Prichard R. A dyf-7 haplotype causes sensory neuron defects and is associated with macrocyclic lactone resistance worldwide in the nematode parasite Haemonchus contortus. Int J Parasitol. 2014;44:1063-71.

14. Elmahalawy ST, Halvarsson P, Skarin M, Höglund J. Genetic variants in dyf-7 validated by droplet digital PCR are not drivers for ivermectin resistance in Haemonchus contortus. Int J Parasitol Drugs Drug Resist. 2018;8:278-86.

15. Laing R, Maitland K, Lecová L, Skuce PJ, Tait A, Devaney E. Analysis of putative resistance gene loci in UK field populations of Haemonchus contortus after 6 years of macrocyclic lactone use. Int $\mathrm{J}$ Parasitol. 2016;46:621-30.

16. Rezansoff AM, Laing R, Gilleard JS. Evidence from two independent backcross experiments supports genetic linkage of microsatellite $\mathrm{Hcms} 8 \mathrm{a} 20$, but not other candidate loci, to a major ivermectin resistance locus in Haemonchus contortus. Int J Parasitol. 2016;46:653-61.

17. Martin F, Dube F, Karlsson Lindsjö O, Eydal M, Höglund J, Bergström TF, et al. Transcriptional responses in Parascaris univalens after in vitro exposure to ivermectin, pyrantel citrate and thiabendazole. Parasit Vectors. 2020;13:342.

18. Xu D, Zhang Y, Zhang Y, Wu Q, Guo Z, Xie W, et al. Transcriptome profiling and functional analysis suggest that the constitutive overexpression of four cytochrome P450s confers resistance to abamectin in Tetranychus urticae from China. Pest Manag Sci. 2021;77:1204-13. 
19. Van Wyk JA, Malan FS. Resistance of field strains of Haemonchus contortus to ivermectin, closantel, rafoxanide and the benzimidazoles in South Africa. Vet Rec. 1988;123:226-8.

20. Coles GC, Bauer C, Borgsteede FHM, Geerts S, Klei TR, Taylor MA, et al. World Association for the Advancement of Veterinary Parasitology (WAAVP) methods for the detection of anthelmintic resistance in nematodes of veterinary importance. Vet Parasitol. 1992;44:35-44.

21. Kotze AC, O’Grady J, Emms J, Toovey AF, Hughes S, Jessop P, et al. Exploring the anthelmintic properties of Australian native shrubs with respect to their potential role in livestock grazing systems. Parasitology. 2009;136:1065-80.

22. Raza A, Lamb J, Chambers M, Hunt PW, Kotze AC. Larval development assays reveal the presence of sub-populations showing high and low-level resistance in a monepantel (Zolvix®)-resistant isolate of Haemonchus contortus. Vet Parasitol. 2016;220:77-82.

23. Demeler J, Gill JH, von Samson-Himmelstjerna G, Sangster NC. The in vitro assay profile of macrocyclic lactone resistance in three species of sheep trichostrongyloids. Int J Parasitol Drugs Drug Resist. 2013;3:109-18.

24. Williamson SM, Wolstenholme AJ. P-glycoproteins of Haemonchus contortus. development of realtime PCR assays for gene expression studies. J Helminthol. 2012;86:202-8.

25. Kotze AC, Prichard RK. Anthelmintic resistance in Haemonchus contortus: history, mechanisms and diagnosis. Adv Parasitol. 2016;93:397-428.

26. Falzon LC, Menzies PI, Shakya KP, Jones-Bitton A, Vanleeuwen J, Avula J, et al. Anthelmintic resistance in sheep flocks in Ontario, Canada. Vet Parasitol. 2013;193:150-62.

27. Veríssimo CJ, Niciura SC, Alberti AL, Rodrigues CF, Barbosa CM, Chiebao DP, et al. Multidrug and multispecies resistance in sheep flocks from São Paulo state, Brazil. Vet Parasitol. 2012;187:20916.

28. Papadopoulos E, Gallidis E, Ptochos S. Anthelmintic resistance in sheep in Europe: a selected review. Vet Parasitol. 2012;189:85-8.

29. Playford MC, Smith AN, Love S, Besier RB, Kluver P, Bailey JN. Prevalence and severity of anthelmintic resistance in ovine gastrointestinal nematodes in Australia (2009-2012). Aust Vet J. 2014;92:464-71.

30. Tsotetsi AM, Njiro S, Katsande TC, Moyo G, Baloyi F, Mpofu J. Prevalence of gastrointestinal helminths and anthelmintic resistance on small-scale farms in Gauteng Province, South Africa. Trop Anim Health Prod. 2013;45:751-61.

31. Yuan W, Lu K, Li H, Liu J, He C, Feng J, et al. Seasonal dynamics of gastrointestinal nematode infections of goats and emergence of ivermectin resistance in Haemonchus contortus in Hubei province, China. Acta Parasitol. 2019;64:638-44.

32. Ballesteros C, Tritten L, O'Neill M, Burkman E, Zaky WI, Xia J, et al. The effects of ivermectin on Brugia malayi females in vitro: a transcriptomic approach. PLoS Negl Trop Dis. 2016 Aug 16;10:e0004929. 
33. Blackhall WJ, Pouliot JF, Prichard RK, Beech RN. Haemonchus contortus: selection at a glutamategated chloride channel gene in ivermectin- and moxidectin-selected strains. Exp Parasitol. 1998;90:42-8.

34. Williamson SM, Storey B, Howell S, Harper KM, Kaplan RM, Wolstenholme AJ. Candidate anthelmintic resistance-associated gene expression and sequence polymorphisms in a tripleresistant field isolate of Haemonchus contortus. Mol Biochem Parasitol. 2011;180:99-105.

35. Rezansoff AM, Laing R, Martinelli A, Stasiuk S, Redman E, Bartley D. The confounding effects of high genetic diversity on the determination and interpretation of differential gene expression analysis in the parasitic nematode Haemonchus contortus. Int J Parasitol. 2019;49:847-58.

36. Martin RJ, Robertson AP, Choudhary S. Ivermectin: an anthelmintic, an insecticide, and much more. Trends Parasitol. 2021;37:48-64.

37. Hernando G, Bouzat C. Caenorhabditis elegans neuromuscular junction: GABA receptors and ivermectin action. PLoS One. 2014;9:e95072.

38. Abongwa M, Buxton SK, Robertson AP, Martin RJ. Curiouser and Curiouser: The macrocyclic lactone, abamectin, is also a potent inhibitor of pyrantel/tribendimidine nicotinic acetylcholine receptors of gastrointestinal worms. PLoS One. 2016;11:e0146854.

39. Khan S, Nisar A, Yuan J, Luo X, Dou X, Liu F, et al. A whole genome re-sequencing based GWA analysis reveals candidate genes associated with ivermectin resistance in Haemonchus contortus. Genes (Basel). 2020;11:367.

40. Xu M, Molento M, Blackhall W, Ribeiro P, Beech R, Prichard R. Ivermectin resistance in nematodes may be caused by alteration of P-glycoprotein homolog. Mol Biochem Parasitol. 1998;91:327-35.

41. Lloberas M, Alvarez L, Entrocasso C, Virkel G, Ballent M, Mate L, et al. Comparative tissue pharmacokinetics and efficacy of moxidectin, abamectin and ivermectin in lambs infected with resistant nematodes: impact of drug treatments on parasite P-glycoprotein expression. Int J Parasitol Drugs Drug Resist. 2012;3:20-7.

42. Matoušková P, Vokřál I, Lamka J, Skálová L. The role of xenobiotic-metabolizing enzymes in anthelmintic deactivation and resistance in helminths. Trends Parasitol. 2016;32:481-91.

43. Kellerová P, Matoušková P, Lamka J, Vokřál I, Szotáková B, Zajíčková M, et al. Ivermectin-induced changes in the expression of cytochromes P450 and efflux transporters in Haemonchus contortus female and male adults. Vet Parasitol. 2019;273:24-31.

44. Vokřál I, Jedličková V, Jirásko R, Stuchlíková L, Bártíková H, Skálová L, et al. The metabolic fate of ivermectin in host (Ovis aries) and parasite (Haemonchus contortus). Parasitology. 2013;140:361-7.

45. Lindblom TH, Dodd AK. Xenobiotic detoxification in the nematode Caenorhabditis elegans. J Exp Zool A Comp Exp Biol. 2006;305:720-30.

46. Laing ST, Ivens A, Butler V, Ravikumar SP, Laing R, Woods DJ, et al. The transcriptional response of Caenorhabditis elegans to ivermectin exposure identifies novel genes involved in the response to reduced food intake. PLoS One. 2012;7:e31367. 
47. Wang T, Nie S, Ma G, Korhonen PK, Koehler AV, Ang CS, et al. The developmental lipidome of Haemonchus contortus. Int J Parasitol. 2018;48:887-95.

48. Pallarés-Trujillo J, López-Soriano FJ, Argilés JM. Lipids: A key role in multidrug resistance? (Review). Int J Oncol. 2000;16:783-98.

49. Kopecka J, Trouillas P, Gašparović A, Gazzano E, Assaraf YG, Riganti C. Phospholipids and cholesterol: inducers of cancer multidrug resistance and therapeutic targets. Drug Resist Updat. 2020;49:100670.

50. Smus JP, Ludlow E, Dallière N, Luedtke S, Monfort T, Lilley C, et al. Coherent anti-Stokes Raman scattering (CARS) spectroscopy in Caenorhabditis elegans and Globodera pallida: evidence for an ivermectin-activated decrease in lipid stores. Pest Manag Sci. 2017;73:2550-8.

51. Ballesteros C, Tritten L, O'Neill M, Burkman E, Zaky WI, Xia J, et al. The effect of in vitro cultivation on the transcriptome of adult Brugia malayi. PLoS Negl Trop Dis. 2016b;10:e0004311.

52. Wong D, Bazopoulou D, Pujol N, Tavernarakis N, Ewbank JJ. Genome-wide investigation reveals pathogen-specific and shared signatures in the response of Caenorhabditis elegans to infection. Genome Biol. 2007;8:R194.

53. Coolon JD, Jones KL, Todd TC, Carr BC, Herman MA. Caenorhabditis elegans genomic response to soil bacteria predicts environment-specific genetic effects on life history traits. PLoS Genet. 2009;5:e1000503.

54. Shin H, Lee H, Fejes AP, Baillie DL, Koo HS, Jones SJ. Gene expression profiling of oxidative stress response of $C$. elegans aging defective AMPK mutants using massively parallel transcriptome sequencing. BMC Res Notes. 2011;4:34.

55. Smith H, Campbell WC. Effect of ivermectin on Caenorhabditis elegans larvae previously exposed to alcoholic immobilization. J Parasitol. 1996;82:187-8.

56. Dent JA, Smith MM, Vassilatis DK, Avery L. The genetics of ivermectin resistance in Caenorhabditis elegans. Proc Natl Acad Sci U S A. 2000;97:2674-9.

57. Harder A. The biochemistry of Haemonchus contortus and other parasitic nematodes. Adv Parasitol. 2016;93:69-94.

\section{Figures}




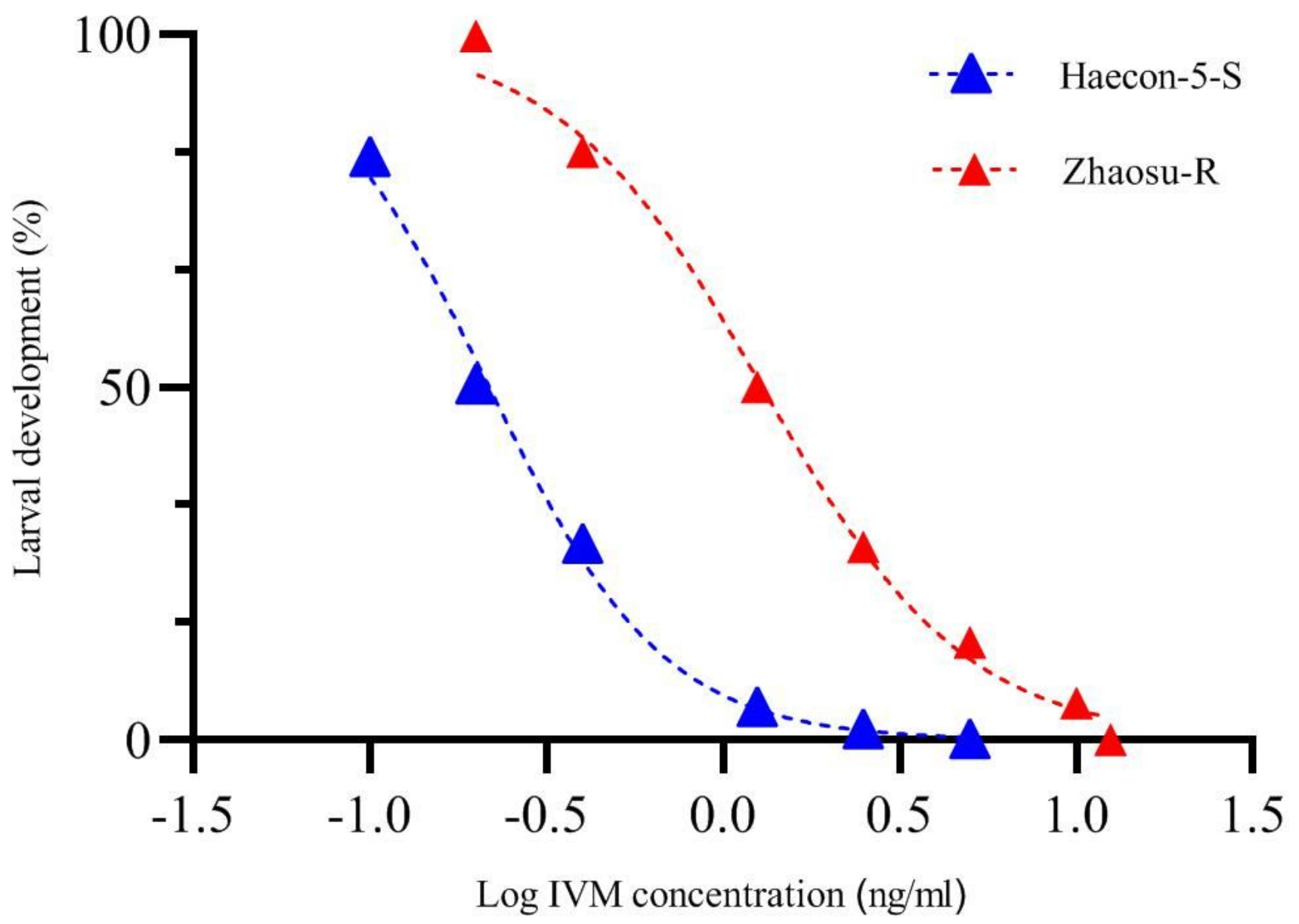

Figure 1

Dose responses for the Haecon-5 and Zhaosu strain of Haemonchus contortus towards ivermectin. Data of each point from three separate experiments with triplicate (mean $\pm \mathrm{SE}$ ). 


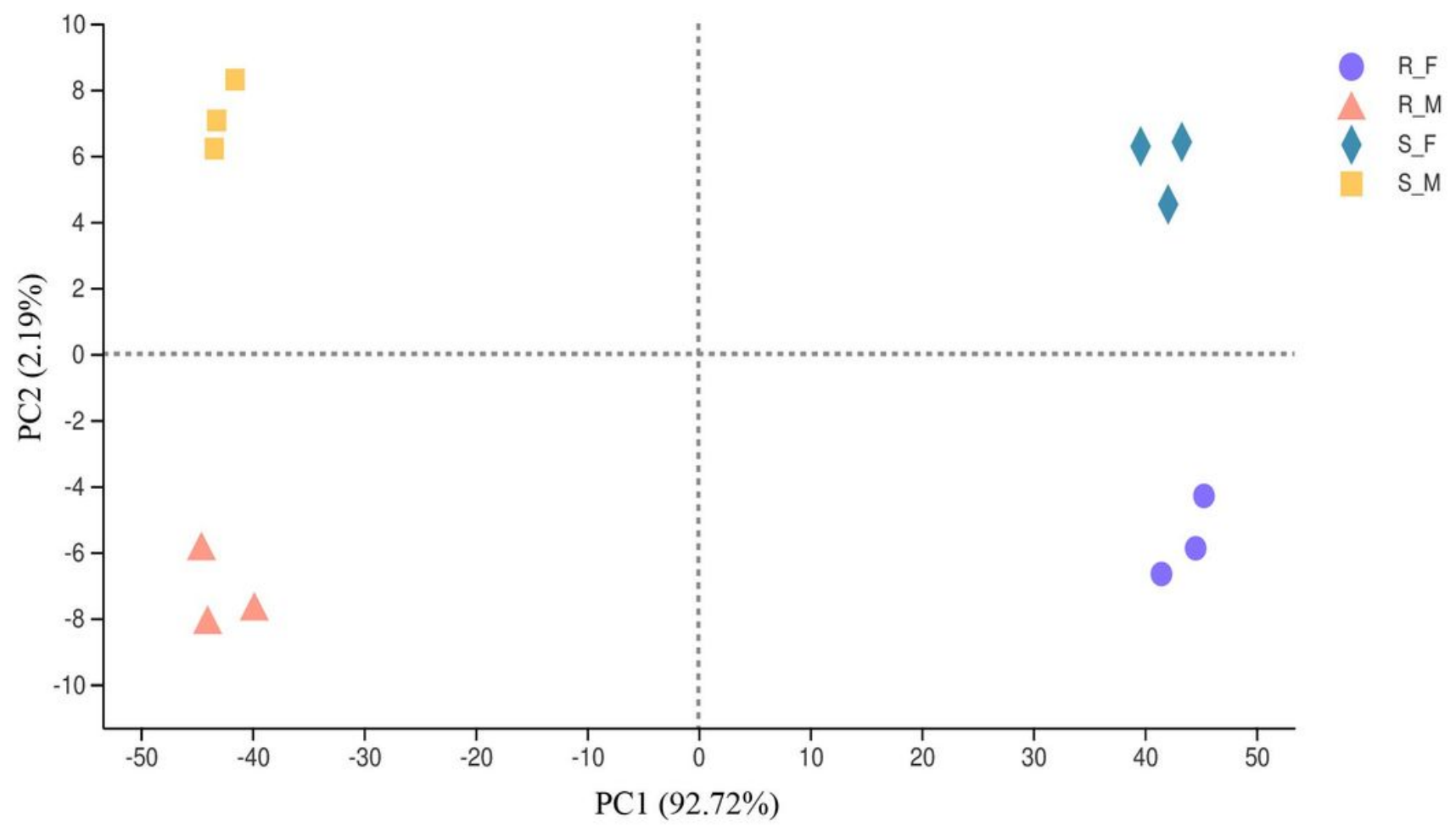

\section{Figure 2}

PCA analysis of the libraries constructed from adult female and male worms of Haemonchus contortus IVM susceptible and resistant strains. Purple circle, resistance female (RF); Orange triangle, resistance male (RM); Blue Diamond, susceptible female (SF); Yellow square, susceptible male (SM).

\section{Figure 3}

DEGs analysis of male and female worms between IVM susceptible and resistance strains of Haemonchus contortus. a Venn diagram shows common and unique DEGs of the male and female; $\mathbf{b}, \mathbf{c}$ Volcano plot of DEGs of male and female between IVM susceptible and resistant strains. Red, green and blue dots represent upregulated, downregulated, and no significant differences genes, respectively.

\section{Figure 4}

Cluster heat map showing the level of expression of genes differentially expressed in male and female worms between IVM susceptible and resistant strains of Haemonchus contortus. $\mathbf{a}$, b Heat map of DEGs of the male and female worms between IVM susceptible and resistant strain. 
b

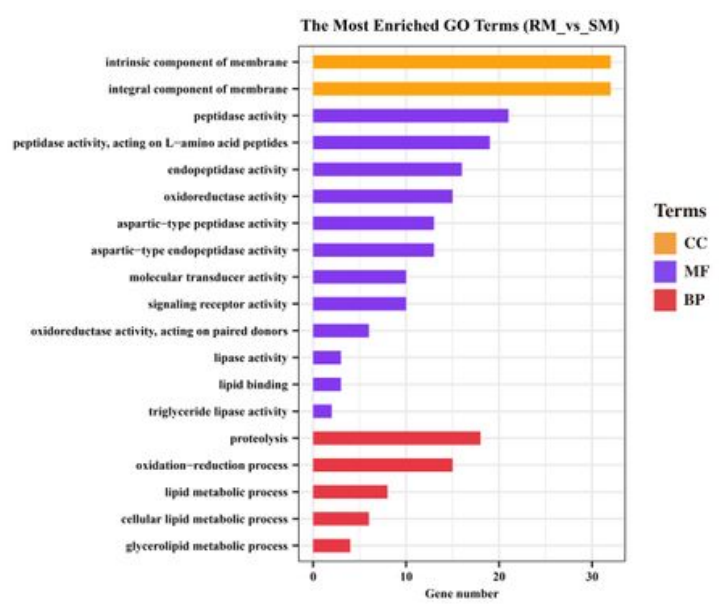

c

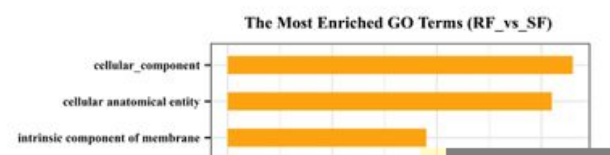

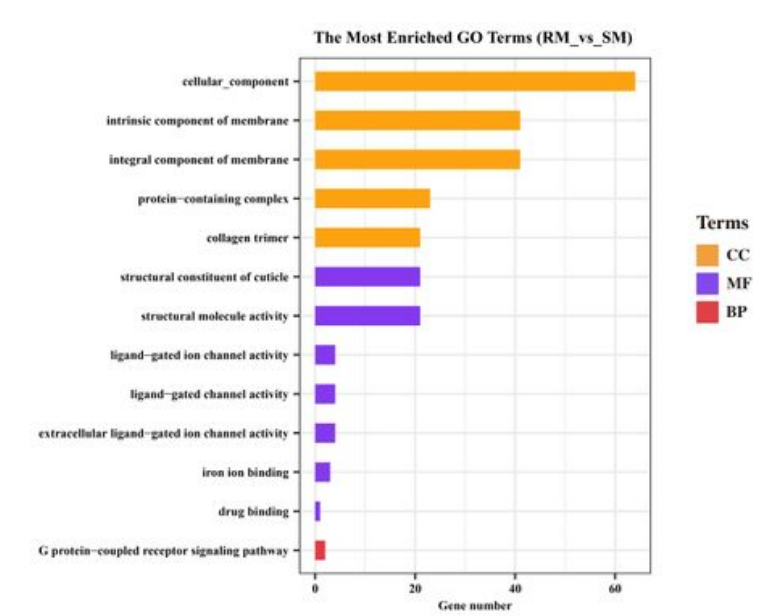

d

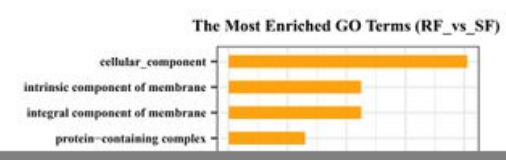

Figure 5

GO classifications of up and down regulated DEGs in male and female worms between IVM susceptible and resistant strains of Haemonchus contortus. $\mathbf{a}, \mathbf{b} \mathrm{GO}$ classifications of up and down regulated genes of male worms between IVM resistant and susceptible strain; $\mathbf{c}, \mathbf{b}$ GO classifications of up and down regulated genes of female worms between IVM resistant and susceptible strain. Y-axis represented GO terms; $\mathrm{X}$-axis represented the gene number. 
a

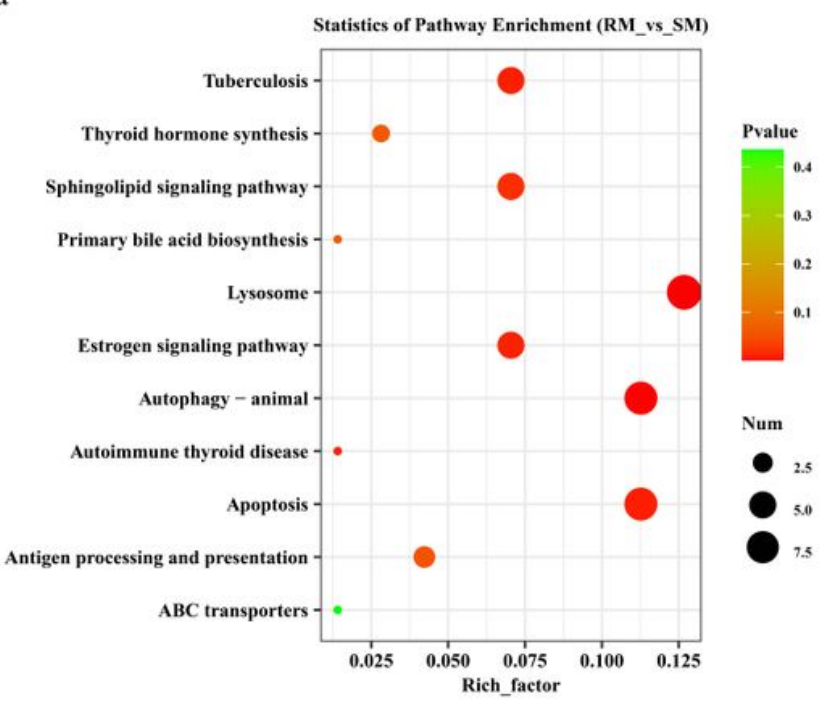

$\mathrm{c}$

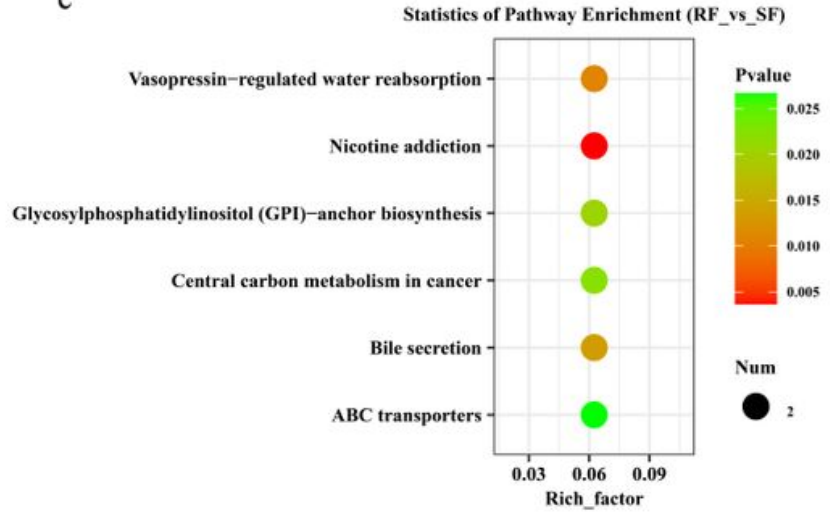

b

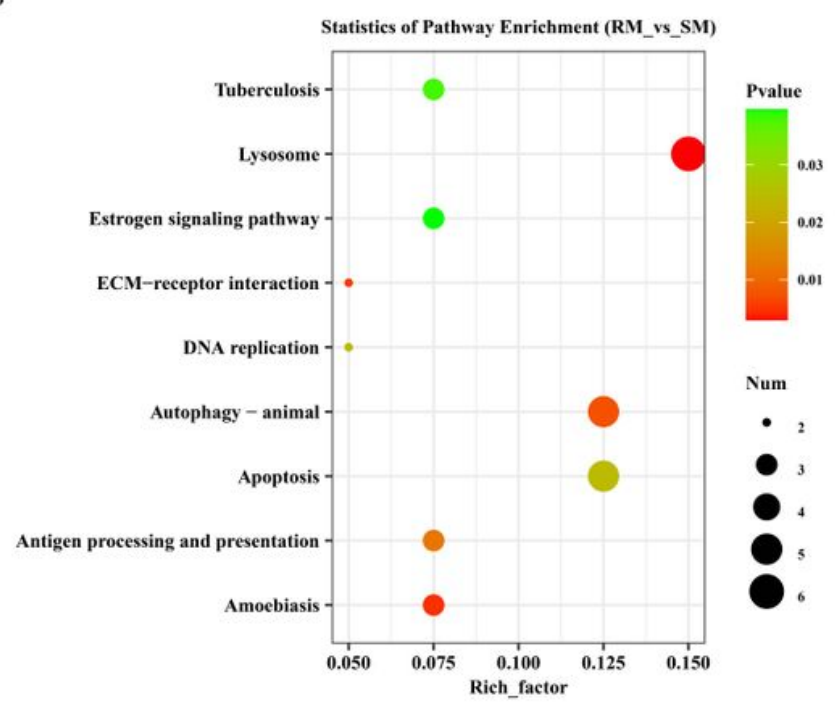

d

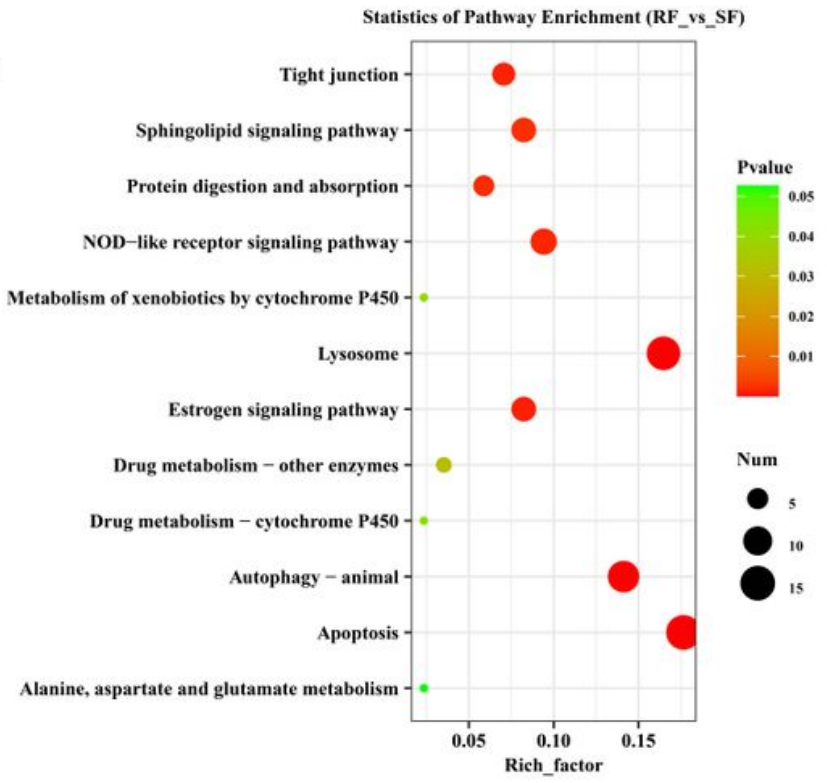

\section{Figure 6}

KEGG enrichment analysis of up and down regulated DEGs in male and female worms between IVM resistant and susceptible strains of Haemonchus contortus. $\mathbf{a}, \mathbf{b}$ The most enriched KEGG pathway of up and down regulated DEGs of male worms between IVM resistant and susceptible strain; $\mathbf{c}$, $\mathbf{d}$ The most enriched KEGG pathway of up and down regulated DEGs of female worms between IVM resistant and susceptible strain. The $X$ and $Y$ axis represents rich factor and pathways. The size and color of the circle represent the number of genes and $p$ value. 
a
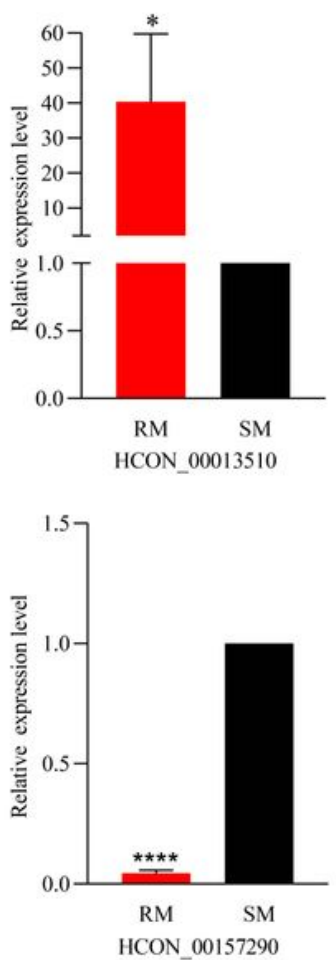

b

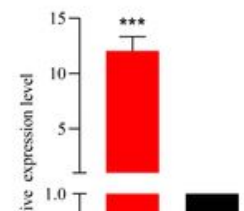

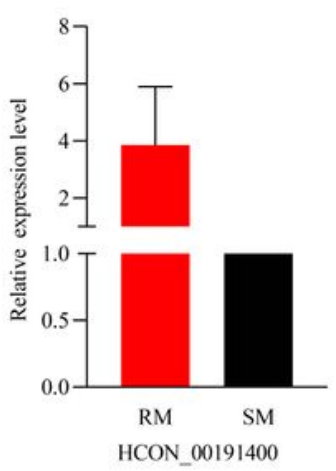
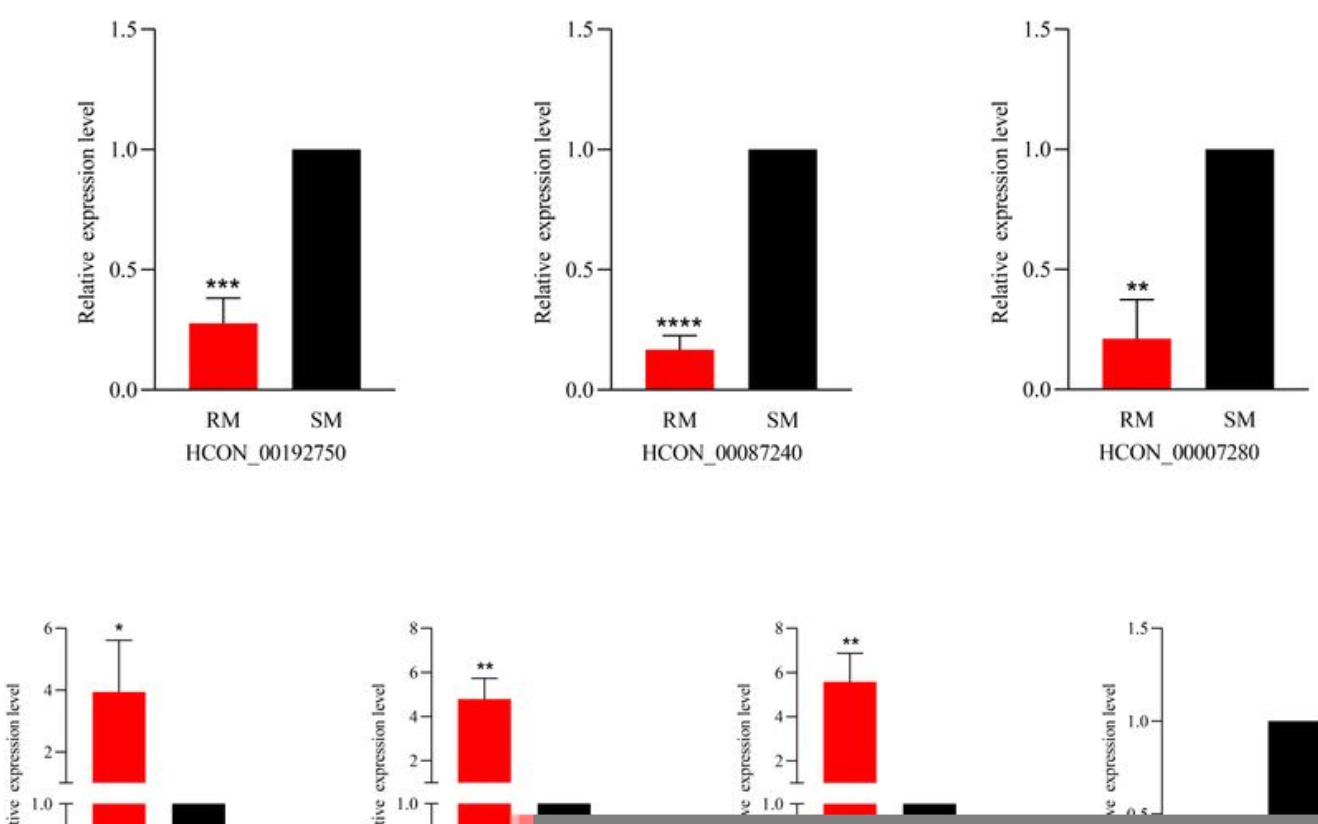

Figure 7

Relative transcript levels of selected genes differentially expressed in male and female worms between IVM susceptible and resistant strains of Haemonchus contortus. $\mathbf{a}, \mathbf{b}$ The validation of DEGs of male and worms between IVM susceptible and resistant strain. Data from the qRT-PCR represent the mean of three

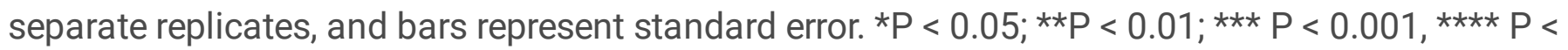
0.0001. 


\section{Supplementary Files}

This is a list of supplementary files associated with this preprint. Click to download.

- graphicalabstract.jpg.png

- Additionalfile1.docx 